\title{
Ordering of anisotropic polarizable polymer chains on the full many-body level
}

\author{
David S. Dean ${ }^{1,2}$ and Rudolf Podgornik ${ }^{2,3,4}$ \\ ${ }^{1}$ Université de Bordeaux and CNRS, Laboratoire Ondes et Matière d'Aquitaine (LOMA), \\ UMR 5798, F-33400 Talence, France \\ ${ }^{2}$ Laboratoire de Physique Théorique (IRSAMC), Université de Toulouse, \\ UPS and CNRS, F-31062 Toulouse, France \\ ${ }^{3}$ Department of Theoretical Physics, J. Stefan Institute, SI-1000 Ljubljana, Slovenia \\ ${ }^{4}$ Department of Physics, Faculty of Mathematics and Physics, University of Ljubljana, \\ SI-1000 Ljubljana, Slovenia
}

(Received 27 December 2011; accepted 27 March 2012; published online 19 April 2012)

\begin{abstract}
We study the effect of dielectric anisotropy of polymers on their equilibrium ordering within meanfield theory, but with a formalism that takes into account the full n-body nature of van der Waals (vdW) forces. Dielectric anisotropy within polymers is to be expected as the electronic properties of the polymer will typically be different along the polymer than across its cross section. It is therefore physically intuitive that larger charge fluctuations can be induced along the chain than perpendicular to it. We show that this dielectric anisotropy leads to n-body interactions which can induce an isotropic-nematic transition. The two body and three body components of the full vdW interaction are extracted and it is shown how the two body term behaves like the phenomenological self-aligningpairwise nematic interaction. At the three body interaction level we see that the nematic phase that is energetically favorable is discotic, however, on the full n-body interaction level we find that the normal axial nematic phase is always the stable ordered phase. The n-body nature of our approach also shows that the key parameter driving the nematic-isotropic transition is the bare persistence length of the polymer chain. ( 2012 American Institute of Physics. [http://dx.doi.org/10.1063/1.3703762]
\end{abstract}

\section{INTRODUCTION}

Semi-flexible polymers, ${ }^{1}$ just like rod-like anisotropic nematogens, form orientationally ordered liquid crystalline mesophases, spanning the concentration regime of chiral nematics, ${ }^{2}$ and all the way to line hexatics. ${ }^{3}$ Among the most important examples of semi-flexible polymer ordering is the double stranded DNA molecule, ${ }^{4,5}$ which makes a plethora of liquid crystalline phases in aqueous solutions. These ordered phases of DNA aqueous dispersions are pertinent to the construction of DNA nano-assemblies ${ }^{6}$ and have recently found a promising new application in the form of virus-like particles. ${ }^{7}$

A rigorous theory of nematic ordering of long rigid nematogens, in dilute solutions, was first proposed in a seminal work by Onsager. ${ }^{8}$ His approach was later adapted to semiflexible polymers by Semenov and Khokhlov, ${ }^{9}$ and within a different formal background by Ronca and Yoon. ${ }^{10}$ A fieldtheoretical reformulation of the nematic-isotropic transition for semi-flexible polymers was then pursued by Gupta and Edwards, ${ }^{1}$ as well as by Tkachenko and Rabin and others. ${ }^{11,12}$ These approaches are all based on the assumption of pairwise additivity of the interaction potential, which is assumed to be of a general nematic form stemming from the Onsager steric interaction. For short range interactions this is of course a reasonable assumption. However, it is well known that electrostatic as well as van der Waals (vdW) interactions usually engender long range non-pairwise additive interactions that in general do not conform to this approximation. ${ }^{13}$
Furthermore, polymers in general show an anisotropic dielectric response, so that the dielectric functions along the axis and perpendicular to the axis differ. This is nicely seen, e.g., in the case of DNA, ${ }^{14}$ where the amplitude of the dielectric function in the axial and the radial directions are very different because of the strong optical anisotropy of DNA. ${ }^{15}$ This dielectric anisotropy has important consequences, as $\mathrm{vdW}$ interactions between anisotropic media lead, in general, to torques induced by electromagnetic field fluctuations, as first realized by Weiss and Parsegian. ${ }^{17,18}$ The details of this effect are complicated, but it persists not only in the non-retarded but also in the retarded limit. ${ }^{19-21}$

The existence of anisotropic vdW interactions now poses a question about their possible role in ordering transitions. While the fundamental importance of the vdW force for the gas-liquid transition is well recognized, we now investigate the possible role of anisotropic vdW interactions for orientational ordering transitions. As it is not clear off-hand whether the effect is significant, it is of utmost importance that the many-body pairwise non-additive nature of vdW interactions be fully treated in the theoretical framework. We thus formulate the theory of orientational ordering of semi-flexible polymers due to anisotropic vdW interactions in a way that allows for a complete and exact re-summation of vdW interaction energy to all levels, including pairs, triplets, quadruplets, etc. Whatever the conclusions of this theory, they can certainly not be undermined by the improper treatment of the n-body aspects of the vdW effect. 
In order to assess the effect of anisotropic vdW interactions, we analyze a rather simple case of semi-flexible polymer ordering driven exclusively by these interactions. We thus study the effects beyond the usual Onsager steric anisotropy ansatz. However, the most fundamental difference with previous studies is that we calculate exactly the contribution of vdW interactions to all orders within the mean-field approximation. Usually, vdW interactions are treated on a pairwise level, or possibly three body Axilrod-Teller level, ${ }^{22}$ but never more. We propose an analytical approach that allows us to sum the vdW interactions to all orders and solve the corresponding mean-field theory exactly. It should be noted that the long range nature of $\mathrm{vdW}$ interactions implies that our meanfield treatment should be reasonably accurate, especially in dense systems. Pursuing this approach we show that, indeed, anisotropic vdW interactions can engender an orientational ordering transition in a solution of stiff polymer chains.

\section{MEAN FIELD VDW FREE ENERGY}

To begin this section we discuss the formulation of vdW forces in terms of dipoles in the system. This approach ultimately allows a general formulation in terms of local dielectric constants which are accessible experimentally. The approach is rather standard, however, we go over it here for the sake of completeness and because our final results will depend on a microscopic cut-off. The exposition given here allows us to determine the microscopic origin of the cut-off and hence estimate its order of magnitude.

The electrostatic energy of of system of interacting dipoles can be written as

$$
H_{E S}=\frac{1}{2} \sum_{\alpha \neq \beta} \mathbf{p}_{\alpha} T_{\alpha \beta}\left(\mathbf{x}_{\alpha}-\mathbf{x}_{\beta}\right) \mathbf{p}_{\beta},
$$

where the dipole-dipole interaction between dipoles $\mathbf{p}_{\alpha}$ and $\mathbf{p}_{\beta}$ at sites $\mathbf{x}_{\alpha}$ and $\mathbf{x}_{\beta}$ can be written as

$$
T_{i j}\left(\mathbf{x}-\mathbf{x}^{\prime}\right)=\nabla_{\mathbf{x}_{i}} \nabla_{\mathbf{x}_{j}^{\prime}} G\left(\mathbf{x}-\mathbf{x}^{\prime}\right),
$$

where $G$ is the Green's function obeying

$$
\varepsilon_{0} \nabla^{2} G(\mathbf{x})=-\delta(\mathbf{x}) .
$$

The vdW part of the partition function can be written in terms of a trace over the dipole degrees of freedom (for a given configuration of the molecules bearing the dipoles) as

$$
Z_{v d W}=\operatorname{Tr} \exp \left(-\beta H_{E S}\right) .
$$

The dipoles can be decoupled by introducing a scalar field $\phi$ and writing

$$
\begin{aligned}
Z_{v d W}= & \int \mathcal{D}[\phi(\mathbf{x})] \operatorname{Tr} \exp \left(-\frac{\beta \varepsilon_{0}}{2} \int\left(\nabla \phi(\mathbf{x})^{2}\right) d \mathbf{x}\right. \\
& \left.+i \beta \sum_{\alpha} \nabla \phi\left(\mathbf{x}_{\alpha}\right) \cdot \mathbf{p}_{\alpha}\right) .
\end{aligned}
$$

In principle, we have added a divergent self-energy of the interaction between a dipole with itself, however, this term is independent of the interaction between different dipoles and the divergence can be regularized.
The term $-i \mathbf{p}_{\alpha} \cdot \nabla \phi\left(\mathbf{x}_{\alpha}\right)$ can be identified as $-\mathbf{p}_{\alpha} \cdot \mathbf{E}\left(\mathbf{x}_{\alpha}\right)$ which is the interaction of the dipole and the local electric field, so that we can thus write $\phi=-i \psi$ where $\psi$ is the local electrostatic potential. As mentioned above, the results obtained in this approach will be regulated by a cut-off and at this point we will explain the origin of the cut-off within the context of our model. A dipole moment is a pair of charges $\pm q_{d}$ separated by a distance $a_{0}$ (but which can vary in size in the presence of the field). The dipole will not interact with fields which oscillate in space over distances shorter than the microscopic scale $a_{0}$. Thus, on scales smaller that $a_{0}$ the medium will behave as vacuum. Therefore, we will regularize our results by restricting the functional integral over $\phi$ in Eq. (5) to fields $\phi$ which have no Fourier component for modes $\mathbf{k}$ such that $|\mathbf{k}|>2 \pi / a_{0}$.

As we are only interested in linear dielectric response models, ignoring completely any higher order saturation effects, we can use the approximation, for each $\mathbf{p}_{\alpha}$, that

$$
\begin{aligned}
\operatorname{Tr} \exp \left(i \beta \nabla \phi(\mathbf{x}) \cdot \mathbf{p}_{\alpha}\right) \simeq & \operatorname{Tr}\left[1+i \beta \nabla_{i} \phi(\mathbf{x}) p_{\alpha i}\right. \\
& \left.-\frac{\beta^{2}}{2} p_{\alpha i} p_{\alpha j} \nabla_{i} \phi(\mathbf{x}) \nabla_{j} \phi(\mathbf{x})\right] .
\end{aligned}
$$

Now if we take the trace over the dipole to be normalized so that $\operatorname{Tr} 1=1$, and use the fact that in the absence of an external field $\operatorname{Tr} p_{\alpha i}=0$, we can write

$$
\operatorname{Tr} \exp \left(i \beta \nabla \phi\left(\mathbf{x}_{\alpha}\right) \cdot \mathbf{p}_{\alpha}\right) \simeq 1-\frac{\beta^{2}}{2} \chi_{i j}^{(\alpha)} \nabla_{i} \phi\left(\mathbf{x}_{\alpha}\right) \nabla_{j} \phi\left(\mathbf{x}_{\alpha}\right),
$$
where $\chi_{i j}^{(\alpha)}=\left\langle p_{\alpha i} p_{j \alpha}\right\rangle$ for a single dipole in the absence of an electric field. Here $\chi_{i j}^{(\alpha)}$ can depend on the local environment of the dipole. In a polymer, for example, $\chi_{i j}$ will be different in directions parallel to the polymer and perpendicular to the polymer, for example, typically in the direction tangent to the polymer the polarizability will be larger and this will be an easy axis, i.e., $\chi_{\|}>\chi_{\perp}$. This is certainly what has been observed in recent study. ${ }^{15}$

Re-exponentiating Eq. (7) for each dipole then gives the result

$$
\begin{aligned}
Z_{v d W}= & \int \mathcal{D}[\phi(\mathbf{x})] \operatorname{Tr} \exp \left(-\frac{\beta \varepsilon_{0}}{2} \int\left(\nabla \phi(\mathbf{x})^{2}\right) d \mathbf{x}\right. \\
& \left.-\frac{\beta^{2}}{2} \sum_{\alpha} \chi_{i j}^{(\alpha)} \nabla_{i} \phi\left(\mathbf{x}_{\alpha}\right) \nabla_{j} \phi\left(\mathbf{x}_{\alpha}\right)\right) .
\end{aligned}
$$

Now assuming the above sum is over lattice sites of the same typical size $a_{0}$ as the dipoles, we can approximate the sum in the above as an integral to obtain

$$
\sum_{\alpha} \chi_{i j}^{(\alpha)} \nabla_{i} \phi\left(\mathbf{x}_{\alpha}\right) \nabla_{j} \phi\left(\mathbf{x}_{\alpha}\right)=\int d \mathbf{x} \chi_{i j}(\mathbf{x}) \nabla_{i} \phi(\mathbf{x}) \nabla_{j} \phi(\mathbf{x}),
$$

where $\chi_{i j}(\mathbf{x})=\chi_{i j}^{(\alpha)} / a_{0}^{3}$. We can thus make the identification of the local dielectric tensor as

$$
\varepsilon_{i j}(\mathbf{x})=\varepsilon_{0} \delta_{i j}+\beta \chi_{i j}(\mathbf{x}) .
$$




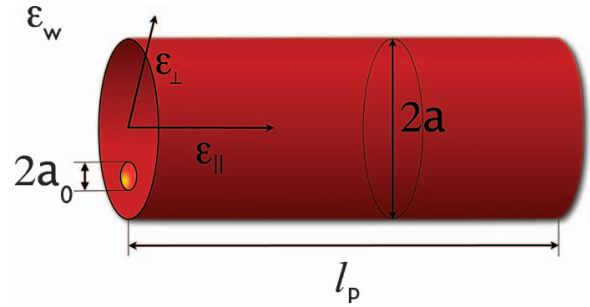

FIG. 1. Schematic representation of the dielectric polymer model with persistence length $l_{p}$. The external medium is water with dielectric constant $\varepsilon_{w}$. The polymer is modeled as a tube with dielectric tensor $\varepsilon_{\|}$in the direction of the tube's local axis of symmetry and $\varepsilon_{\perp}$ perpendicular to the axis of symmetry. The diameter of the tube $a$ is taken to be larger than the atomic/molecular size $a_{0}$ which represents the length of dipoles present in the composite molecules or atoms.

The chain is then assumed to be composed of an anisotropic macroscopic dielectric material that has a dielectric response function $\varepsilon_{\|}$and $\varepsilon_{\perp}$, respectively, parallel and perpendicular to the chain. A schematic for the model is shown in Fig. 1. The model we adopt for the polymer is that of a continuous cylindrical chain. We denote the trajectory of the centre of the polymer about its axis of cylindrical symmetry to be within the arc-length parameterization $\mathbf{x}(s)$, so that the tangent vector defined as

$$
\mathbf{t}(s)=\dot{\mathbf{x}}(s)
$$

is normalized such that $\mathbf{t}^{2}(s)=1$. We now use the matrix $p_{i j}$ $=t_{i} t_{j}$ to project onto the direction parallel to the polymer and the matrix $q_{i j}=\delta_{i j}-t_{i} t_{j}$ to project onto the plane perpendicular to the polymer. Putting all this together gives the partition function for the vdW interactions as

$$
Z_{v d W}=\int \mathcal{D}[\phi(\mathbf{x})] \exp \left(-\beta \mathcal{E}_{E S}\right),
$$

where

$$
\begin{aligned}
\mathcal{E}_{E S}= & \frac{1}{2} \varepsilon_{w} \int_{V_{w}}(\nabla \phi(\mathbf{x}))^{2} d \mathbf{x}+\frac{1}{2} \varepsilon_{\|} \int_{V_{p}} t_{i} t_{j} \nabla_{i} \phi(\mathbf{x}) \nabla_{j} \phi(\mathbf{x}) d \mathbf{x} \\
& +\frac{1}{2} \varepsilon_{\perp} \int_{V_{p}}\left(\delta_{i j}-t_{i} t_{j}\right) \nabla_{i} \phi(\mathbf{x}) \nabla_{j} \phi(\mathbf{x}) d \mathbf{x} .
\end{aligned}
$$

Here $V_{w}$ indicates the volume occupied by the solvent, which is assumed to have an isotropic dielectric tensor $\varepsilon_{i j}=\varepsilon_{w} \delta_{i j}$, and $V_{p}$ is the volume occupied by the polymer which has an anisotropic dielectric tensor $\varepsilon_{i j}=\varepsilon_{\|} p_{i j}+\varepsilon_{\perp} q_{i j}$. The above can be written in terms of an integral over $V$ the total volume and $V_{p}$ the volume occupied by the polymer:

$$
\begin{aligned}
\mathcal{E}_{E S}= & \frac{1}{2} \varepsilon_{w} \int_{V}(\nabla \phi(\mathbf{x}))^{2} d \mathbf{x}+\frac{1}{2} \varepsilon_{\|} \int_{V_{p}} t_{i} t_{j} \nabla_{i} \phi(\mathbf{x}) \nabla_{j} \phi(\mathbf{x}) d \mathbf{x} \\
& +\frac{1}{2} \int_{V_{p}}\left(\left(\varepsilon_{\perp}-\varepsilon_{w}\right) \delta_{i j}-\varepsilon_{\perp} t_{i} t_{j}\right) \nabla_{i} \phi(\mathbf{x}) \nabla_{j} \phi(\mathbf{x}) d \mathbf{x} .
\end{aligned}
$$

We now define the indicator function for the surface perpendicular to $\mathbf{t}(s)$ containing points $\mathbf{y}$ closer than $a$ from the central point $\mathbf{x}(s)$, and which therefore contain polymer material. We denote this indicator function by $I_{a}(\mathbf{x}(s), \mathbf{t}(s), \mathbf{y})$. In terms of this function Eq. (14) then becomes

$$
\begin{aligned}
\mathcal{E}_{E S}= & \frac{1}{2} \varepsilon_{w} \int_{V}(\nabla \phi(\mathbf{x}))^{2} d \mathbf{x}+\frac{1}{2} \int_{0}^{L} d s d \mathbf{y} I_{a}(\mathbf{x}(s), \mathbf{t}(s), \mathbf{y}) \\
& \times\left[\left(\varepsilon_{\perp}-\varepsilon_{w}\right) \delta_{i j}+\left(\varepsilon_{\|}-\varepsilon_{\perp}\right) t_{i}(s) t_{j}(s)\right] \nabla_{i} \phi(\mathbf{y}) \nabla_{j} \phi(\mathbf{y}) .
\end{aligned}
$$

The indicator function is explicitly given by

$$
\left.I_{a}(\mathbf{x}(s)), \mathbf{t}(s), \mathbf{y}\right)=\delta([\mathbf{y}-\mathbf{x}(s)] \cdot \mathbf{t}(s)) \theta(a-|\mathbf{y}-\mathbf{x}(s)|),
$$

the first term picks out the surface perpendicular to the polymer direction and in the second term, the Heaviside $\theta$ function, restricts integration to points within the radius $a$ of the polymer. If we introduce the joint density field of $\mathbf{t}$ and $\mathbf{x}$ defined by

$$
\Gamma(\mathbf{x}, \mathbf{t})=\int_{0}^{L} d s \delta(\mathbf{x}-\mathbf{x}(s)) \delta(\mathbf{t}-\mathbf{t}(s)),
$$

the electrostatic energy can be written as

$$
\begin{aligned}
\mathcal{E}_{E S}= & \frac{1}{2} \varepsilon_{w} \int_{V}(\nabla \phi(\mathbf{x}))^{2} d \mathbf{x} \\
& +\frac{1}{2} \int d \mathbf{x} d \mathbf{t} \Gamma(\mathbf{x}, \mathbf{t}) \int d \mathbf{y} I_{a}(\mathbf{x}, \mathbf{t}, \mathbf{y}) \times\left[\left(\varepsilon_{\perp}-\varepsilon_{w}\right) \delta_{i j}\right. \\
& \left.+\left(\varepsilon_{\|}-\varepsilon_{\perp}\right) t_{i} t_{j}\right] \nabla_{i} \phi(\mathbf{y}) \nabla_{j} \phi(\mathbf{y}) .
\end{aligned}
$$

In the mean field approximation, we replace the density field $\Gamma(\mathbf{x}, \mathbf{t})$ by its spatially averaged value

$$
\langle\Gamma(\mathbf{x}, \mathbf{t})\rangle=\frac{1}{V} \int d \mathbf{x} \Gamma(\mathbf{x}, \mathbf{t})=\frac{L}{V} \Omega(\mathbf{t}),
$$

where $\Omega(\mathbf{t})$ is the probability distribution of the tangent vector t. We now use the fact that

$$
\int d \mathbf{x} I_{a}(\mathbf{x}, \mathbf{t}, \mathbf{y})=\pi a^{2},
$$

and define the order parameter matrix $\sigma_{i j}$ as

$$
\int d \mathbf{t} \Omega(\mathbf{t}) t_{i} t_{j}=\left\langle t_{i} t_{j}\right\rangle=\sigma_{i j},
$$

to obtain the mean field expression for the electrostatic energy as

$$
\begin{aligned}
\mathcal{E}_{E S}= & \int_{V} d \mathbf{x} \frac{1}{2}\left[(\nabla \phi(\mathbf{x}))^{2}\left(\varepsilon_{w}+\frac{\pi a^{2} L}{V}\left(\varepsilon_{\perp}-\varepsilon_{w}\right)\right)\right. \\
& \left.+\frac{\pi a^{2} L}{V}\left(\epsilon_{\|}-\epsilon_{\perp}\right) \sigma_{i j} \nabla_{i} \phi(\mathbf{x}) \nabla_{j} \phi(\mathbf{x})\right],
\end{aligned}
$$

or alternatively as

$$
\mathcal{E}_{E S}=\frac{1}{2} \int_{V} d \mathbf{x} \tilde{\varepsilon}_{i j} \nabla_{i} \phi(\mathbf{x}) \nabla_{j} \phi(\mathbf{x}) .
$$

where

$$
\tilde{\varepsilon}_{i j}=\left(\varepsilon_{w}+v\left(\varepsilon_{\perp}-\varepsilon_{w}\right)\right) \delta_{i j}+v\left(\varepsilon_{\|}-\varepsilon_{\perp}\right) \sigma_{i j}
$$

is the effective mean field dielectric constant and where $v=\pi a^{2} L / V$ is the volume fraction of the polymer. Note that the normalization condition $\mathbf{t}^{2}(s)=1$ implies that the order parameter matrix obeys the constraint $\operatorname{Tr} \sigma=1$. Within this mean field approximation the functional integral over the field 
$\phi$ can be evaluated explicitly giving the corresponding free energy

$$
\beta \mathcal{F}_{v d W}=-\ln \left(\mathcal{Z}_{v d W}\right)=\frac{1}{2} V \int \frac{d^{3} \mathbf{k}}{(2 \pi)^{3}} \log \left(\tilde{\varepsilon}_{i j} k_{i} k_{j}\right) .
$$

This is the form of the free energy corresponding to thermal fluctuations of the electrostatic field that we will use later. It is equivalent to the zero Matsubara frequency or static vdW interactions as it is well known. ${ }^{22}$ In the full Lifshitz theory ${ }^{16}$ with assumed complete magnetic homogeneity of the space, the above formula is generalized to

$$
\beta \mathcal{F}_{v d W}=-\ln \left(\mathcal{Z}_{v d W}\right)=V \sum_{n=0}^{\infty} \int \frac{d^{3} \mathbf{k}}{(2 \pi)^{3}} \log \left(\tilde{\varepsilon}_{i j}\left(l \xi_{n}\right) k_{i} k_{j}\right),
$$

where the $n$ summation (the prime indicates that $n=0$ term has a weight of 1/2) is over the imaginary Matsubara frequencies $\xi_{n}=2 \pi n k_{B} T / \hbar$, where $k_{B}$ is the Boltzmann constant, $T$ is the absolute temperature, and $\hbar$ is the Planck constant divided by $2 \pi$.

Here we compute the zero Matsubara frequency vdW free energy, however, this result can then be trivially extended to compute the contribution from other frequencies. We must compute an integral of the form

$$
W(B)=\int d^{3} \mathbf{k} \ln \left(B_{i j} k_{i} k_{j}\right) .
$$

To proceed, we notice that

$$
\frac{\partial W(B)}{\partial B_{p q}}=I_{p q} \int_{0}^{\Lambda} k^{2} d k=I_{p q} \frac{\Lambda^{3}}{3}
$$

where

$$
I_{p q}=\int d \hat{\mathbf{k}} \frac{\hat{k}_{p} \hat{k}_{q}}{B_{i j} \hat{k}_{i} \hat{k}_{j}}
$$

Here $\hat{\mathbf{k}}$ denotes the unit vector and $\Lambda$ is the ultraviolet cut-off corresponding to length scales below which the electromagnetic field fluctuations are cut off. We now note that

$$
\begin{aligned}
J_{p q}(B) & =\int d^{3} \mathbf{k} \exp \left(-\frac{1}{2} \mathbf{k}^{2}\right) \frac{k_{p} k_{q}}{B_{i j} k_{i} k_{j}} \\
& =\int_{0}^{\infty} k^{2} d k \exp \left(-\frac{1}{2} k^{2}\right) I_{p q}\left(B_{i j}\right)=\sqrt{\frac{\pi}{2}} I_{p q}\left(B_{i j}\right),
\end{aligned}
$$

and use the identity

$$
\begin{aligned}
J_{p q}(B) & =\int d^{3} \mathbf{k} \exp \left(-\frac{1}{2} \mathbf{k}^{2}\right) k_{p} k_{q} \int_{0}^{\infty} \frac{d t}{2} \exp \left(-\frac{t}{2} B_{i j} k_{i} k_{j}\right) \\
& =\frac{1}{2}(2 \pi)^{\frac{3}{2}} \int_{0}^{\infty} d t(I+t B)_{p q}^{-1} \operatorname{det}(1+t B)^{-\frac{1}{2}}
\end{aligned}
$$

where $I$ denotes the identity matrix. Putting all of this together we obtain

$$
I_{p q}(B)=2 \pi \int_{0}^{\infty} d t(1+t B)_{p q}^{-1} \operatorname{det}(I+t B)^{-\frac{1}{2}}=\frac{\partial W(B)}{\partial B_{p q}} .
$$

Thus, we derive the effective potential $W(B)$ in the form of

$$
\begin{aligned}
& W(B=I+\gamma \sigma) \\
& =4 \pi \int_{0}^{\infty} \frac{d t}{t}\left[\frac{1}{(1+t)^{\frac{3}{2}}}-\operatorname{det}(I+t B)^{-\frac{1}{2}}\right] \\
& =4 \pi \int_{0}^{\infty} \frac{d t}{t}\left[\frac{1}{(1+t)^{\frac{3}{2}}}-\operatorname{det}(I+t(I+\gamma \sigma))^{-\frac{1}{2}}\right] .
\end{aligned}
$$

Using this result the zero Matsubara frequency vdW part of the free energy can then be written in a compact form as

$$
\begin{aligned}
\frac{\beta \tilde{\mathcal{F}}_{v d W}(\sigma)}{V}= & \frac{\Lambda^{3}}{12 \pi^{2}}\left[\ln \left(\varepsilon_{w}+v\left(\varepsilon_{\perp}-\varepsilon_{w}\right)\right)+\int_{0}^{\infty} \frac{d t}{t}\right. \\
& \left.\times\left(\frac{1}{(1+t)^{\frac{3}{2}}}-\operatorname{det}[I+t(I+\gamma \sigma)]^{-\frac{1}{2}}\right)\right],
\end{aligned}
$$

where

$$
\gamma=\frac{\nu\left(\varepsilon_{\|}-\varepsilon_{\perp}\right)}{\varepsilon_{w}+\nu\left(\varepsilon_{\perp}-\varepsilon_{w}\right)} .
$$

The zero Matsubara frequency vdW free energy may now be expanded in terms of $\gamma$, the expansion up to order $\gamma^{2}$ corresponding to the pairwise approximation for the vdW interaction. Carrying out this expansion to third order in $\gamma$ yields, up to terms independent of $\sigma$,

$$
\begin{aligned}
& \frac{\beta \tilde{\mathcal{F}}_{v d W}(\sigma)}{V} \\
& =\frac{\Lambda^{3}}{12 \pi^{2}}\left[\frac{\gamma}{3} \operatorname{Tr} \sigma-\frac{\gamma^{2}}{30}\left(2 \operatorname{Tr} \sigma^{2}+(\operatorname{Tr} \sigma)^{2}\right)\right. \\
& \left.\quad+\frac{\gamma^{3}}{315}\left(8 \operatorname{Tr} \sigma^{3}+12 \operatorname{Tr} \sigma^{2} \operatorname{Tr} \sigma+(\operatorname{Tr} \sigma)^{3}\right)+\mathcal{O}\left(\gamma^{4}\right)\right] .
\end{aligned}
$$

It is interesting to compare our free energy functional at the order of $\gamma^{2}$ with that derived within the same meanfield approximation for nematic interactions between polymers where the pairwise interaction energy is given by ${ }^{1}$

$$
\mathcal{E}_{N}=\frac{1}{2} u \int_{0}^{L} \int_{0}^{L} d s d s^{\prime}\left(\mathbf{t}(s) \times \mathbf{t}\left(s^{\prime}\right)\right)^{2} \delta\left(\mathbf{x}(s)-\mathbf{x}\left(s^{\prime}\right)\right) .
$$

The form of this interaction makes it energetically favorable for the polymers to align locally in a parallel fashion. In the mean field approximation this gives a $\sigma$ dependent mean-field free energy of nematic interaction

$$
\frac{\beta \tilde{\mathcal{F}}_{N}(\sigma)}{V}=\frac{L^{2}}{2 V} \beta u\left[(\operatorname{Tr} \sigma)^{2}-\operatorname{Tr} \sigma^{2}\right] .
$$

Recalling that as Tr $\sigma=1$ is fixed, we can identify an effective nematic interaction parameter for the vdW interaction by identifying the coefficients of $\operatorname{Tr}^{2}$ in Eqs. (36) and (38) to obtain

$$
u_{e}=\frac{k_{B} T \Lambda^{3} \gamma^{2} V}{90 \pi^{2} L^{2}}
$$


The effective nematic interaction is thus proportional to the square of the relative anisotropy polarizability $\gamma$ and thus crucially depends on the non-isotropic nature of the polymer material. Note also that the sign of the interaction is always positive. This ties up with the fact that polarizable cylinders have a preference to align in a parallel fashion, thus favoring nematic order. However, only stiff molecules with non-isotropic dielectric response, such as DNA or carbon nanotubes, can be expected to exhibit this particular tendency toward nematic ordering.

\section{POLYMER FREE ENERGY}

The polymer chain itself is not featureless and will be modeled within the framework of the semi-flexible KratkyPorod model, where the polymer's conformational energy is given by

$$
\mathcal{E}_{K P}=\frac{1}{2} K \int_{0}^{L} d s \dot{\mathbf{t}}(s)^{2} \quad \text { with the constraint } \quad \mathbf{t}(s)^{2}=1,
$$

and where the last constraint stems from the fixed length of the monomers. Instead of dealing with this constraint locally, which is a difficult task, we simplify the model as suggested by Edwards and Gupta, ${ }^{1}$ by taking it into account globally in the form

$$
\left\langle\mathbf{t}(s)^{2}\right\rangle=1 .
$$

This then leads to the effective partition function of the polymer chain in the form

$$
\mathcal{E}_{K P}=\frac{1}{2} K \int_{0}^{L} \dot{\mathbf{t}}(s)^{2} d s+\frac{1}{2} \lambda \int_{0}^{L}\left(\mathbf{t}(s)^{2}-1\right) d s,
$$

where $\lambda$ is a Lagrange multiplier to be evaluated selfconsistently from the global constraint of Eq. (41). The polymer partition function is then given by

$$
\begin{aligned}
\mathcal{Z}_{K P}= & \int \mathcal{D}[\mathbf{x}(s)] \exp \left(-\beta \mathcal{E}_{K P}[\mathbf{x}(s)]\right) \quad \text { with } \\
& \int \mathcal{D}[\mathbf{x}(s)] \dot{\mathbf{x}}(s)^{2} \exp \left(-\beta \mathcal{E}_{K P}[\mathbf{x}(s)]\right)=\mathcal{Z}_{K P} .
\end{aligned}
$$

In order to couple this model with our mean field calculation of the vdW free energy we need to compute the free energy of the polymer with the constraint that

$$
\left\langle t_{i} t_{j}\right\rangle=\frac{1}{L} \int_{0}^{L} d s \dot{x}_{i}(s) \dot{x}_{j}(s)=\sigma_{i j} .
$$

This average constraint can be imposed using a Fourier representation of the delta function

$$
\begin{aligned}
\delta & \left(\frac{1}{2} \beta L \sigma_{i j}-\frac{1}{2} \beta \int_{0}^{L} d s \dot{x}_{i}(s) \dot{x}_{j}(s)\right) \\
& =\int d s_{i j} \exp \left(\frac{\beta}{2} L s_{i j} \sigma_{i j}-\frac{\beta}{2} \int_{0}^{L} d s s_{i j} \dot{x}_{i}(s) \dot{x}_{j}(s)\right),
\end{aligned}
$$

where the variables $s_{i j}$ are integrated along the imaginary axis. The partition function for the constrained polymer is thus given by

$$
\begin{aligned}
Z_{K P}= & \int \mathcal{D}[\mathbf{x}(s)] d s_{i j} \exp \left(-\frac{\beta K}{2} \int_{0}^{L} d s \ddot{\mathbf{x}}^{2}(s)\right. \\
& -\frac{\beta}{2} \lambda \int_{0}^{L} d s\left(\dot{\mathbf{x}}^{2}(s)-1\right)+\frac{\beta}{2} L s_{i j} \sigma_{i j} \\
& \left.-\frac{\beta}{2} \int_{0}^{L} d s s_{i j} \dot{\mathbf{x}}_{i}(s) \dot{\mathbf{x}}_{j}(s)\right) .
\end{aligned}
$$

In order compute $Z_{K P}$ we first of all introduce the Rouse, or polymer Fourier, decomposition

$$
\mathbf{x}(s)=\int \frac{d \omega}{2 \pi} \mathbf{x}(\omega) e^{-i \omega s} .
$$

The complete polymer part of the partition function can thus be written as

$$
\begin{aligned}
\mathcal{Z}_{K P}= & \int d s_{i j} \exp \left(\frac{1}{2} \beta \lambda L+\frac{1}{2} \beta L s_{i j} \sigma_{i j}\right) \prod_{\omega} \\
& \times \int d \mathbf{x}(\omega) \exp \left(-\beta \tilde{\mathcal{E}}_{P o l}(\mathbf{x}(\omega))\right),
\end{aligned}
$$

where $L$ is the total length of the polymer chain, and

$$
\tilde{\mathcal{E}}_{K P}(\mathbf{x}(\omega))=\frac{1}{2} a_{i j}(\omega) x_{i}(\omega) x^{*}{ }_{j}(\omega),
$$

where the matrix $a(\omega)$ has components

$$
a_{i j}(\omega)=\omega^{2}\left(\left(K \omega^{2}+\lambda\right) \delta_{i j}+s_{i j}\right) .
$$

The functional integral over the Rouse modes is now Gaussian and can be evaluated explicitly yielding, up to irrelevant multiplicative constants,

$$
\mathcal{Z}_{K P}=\exp \left(\frac{\beta}{2} \lambda L+\frac{\beta}{2} L s_{i j} \sigma_{i j}\right) \prod_{\omega}(\operatorname{det}(a(\omega)))^{-1 / 2} .
$$

In the large $L$ limit the corresponding free energy is given by

$$
\begin{aligned}
\beta \tilde{\mathcal{F}}_{K P}= & -\ln \left(Z_{K P}\right)=L \min _{\lambda, s_{i j}} \\
& \times\left\{\frac{1}{4 \pi} \int d \omega \ln (\operatorname{det} a(\omega))-\frac{\beta}{2} \lambda-\frac{\beta}{2} \sigma_{i j} s_{i j}\right\} .
\end{aligned}
$$

The integral over $\omega$ can be evaluated easily in an explicit form in terms of the eigenvalues $s_{\mu}$ of $s$ and $\sigma_{\mu}$ of $\sigma$, yielding up to an overall constant independent of $\sigma$

$$
\beta \tilde{\mathcal{F}}_{K P}=L \min _{\lambda, s_{\mu}}\left\{\frac{1}{4} \sum_{\mu} \sqrt{\frac{\lambda+s_{\mu}}{K}}-\frac{\beta \lambda}{2}-\frac{\beta}{2} \sigma_{\mu} s_{\mu}\right\} .
$$

The minimization is now carried out with respect to fields $s$ and $\lambda$ to yield

$$
\beta \tilde{\mathcal{F}}_{K P}=\frac{L k_{B} T}{32 K} \sum_{\mu} \sigma_{\mu}^{-1}=\frac{L k_{B} T}{32 K} \operatorname{Tr} \sigma^{-1} .
$$

We note that the constraint imposed by $\lambda$ gives that $\operatorname{Tr} \sigma=1$. 


\section{TOTAL FREE ENERGY}

The free energy density per unit volume as a function of the order parameter $\sigma$ is denoted by $\tilde{f}(\sigma)=\tilde{\mathcal{F}}_{T}(\sigma) / V$, and can be expressed, up to terms independent of $\sigma$, as

$$
\begin{aligned}
\beta \tilde{f}(\sigma)= & \frac{\Lambda^{3}}{12 \pi^{2}}\left[\int _ { 0 } ^ { \infty } \frac { d t } { t } \left(\frac{1}{(1+t)^{\frac{3}{2}}}\right.\right. \\
& \left.\left.-\operatorname{det}[I+t(I+\gamma \sigma)]^{-\frac{1}{2}}\right)+c \operatorname{Tr} \sigma^{-1}\right] .
\end{aligned}
$$

The dimensionless parameter $c$ is given by

$$
c=\frac{3}{8} \frac{\pi v}{\Lambda^{3} a^{2} l_{b} b},
$$

where $l_{p}=K / b k_{B} T$ is the dimensionless bare persistence length of the polymer in units of the monomer length $b$. From the definition of $\gamma$ in Eq. (35), it is clear that $0<\gamma$ $<\left(\varepsilon_{\|}-\varepsilon_{\perp}\right) / \varepsilon_{\|}$. This simple fact is a crucial point arising from our analysis. The fact that the effective interaction parameter saturates at high volume concentrations means that a phase transition cannot be induced by simply increasing the concentration of polymer as in the case of models with selfaligning-pairwise interactions. ${ }^{1}$ Physically this result can be interpreted by the fact that n-body interactions tend to frustrate the ordering which is induced by the pairwise term.

The cut-off $\Lambda$ associated with vdW interactions is associated with the breakdown of the continuum dielectric description and is usually taken to be of the atomic size, ${ }^{22}$ this is consistent with our discussion above where we relate the cutoff to the size of dipole moments. We thus set $\Lambda=2 \pi / a_{0}$ and write $a=\lambda a_{0}$ where $\lambda$ is the ratio between the atomic and the macro-molecular sizes $a_{0}$ and $a$, which is thus larger than 1 . This means that we can write

$$
c \simeq \frac{3 v a}{64 \pi^{2} \lambda^{3} b l_{p}}=\frac{3}{64 \pi^{2} \lambda^{3}} \frac{v}{l_{p}}=\mathcal{C} . v
$$

Further, we notice that the precise values of the parameters $a$ and $b$ can be absorbed into a redefinition of the persistence length of the polymer, i.e. $l_{p} \longrightarrow l_{p}(b / a)$. In the analysis that follows, the dimensionless parameter $c$ is expected to be small as by definition $v<1$ and $l_{p}>1$ and $\lambda>1$.

\section{NEMATIC TRANSITION}

The parameter $\gamma$ is small in the limit where $\varepsilon_{\|} \approx \varepsilon_{\perp}$ and/or when the volume fraction $v$ is small. However for strong contrasts in $\varepsilon_{\|}$and $\varepsilon_{\perp}$ it can be of order 1. It is convenient to measure the components of the polymer's dielectric tensor in terms of the dielectric constants of the solvent, we write $\varepsilon_{\|}=\varepsilon_{w} \epsilon_{\|}$and $\varepsilon_{\|}=\varepsilon_{w} \epsilon_{\|}$, which now gives

$$
\gamma=\left(\frac{\epsilon_{\|}-\epsilon_{\perp}}{\epsilon_{\|}-1}\right)\left(1-\frac{1}{1+v\left(\epsilon_{\|}-1\right)}\right) .
$$

Therefore $\gamma$ is a monotonic function of $v$ and the absolute value of $\gamma$ increases upon increasing $\nu$. In order to proceed we write the tensor $\sigma$ as

$$
\sigma=\frac{1}{3}(I+Q)
$$

where $Q$ is the order parameter tensor

$$
Q=\left(\begin{array}{ccc}
-S+T & 0 & 0 \\
0 & -S-T & 0 \\
0 & 0 & 2 S
\end{array}\right)
$$

Here $S$ is the standard uniaxial nematic scalar order parameter and $T$ is the measure of the biaxality.

At the pairwise level, the vdW interaction to order $\gamma^{2}$ can be mapped onto a nematic interaction Eq. (39), a uniaxial nematic alignment of the polymers is favored and indeed Gupta and Edwards ${ }^{1}$ found in their analysis that only these phases are thermodynamically stable. Thus in what follows we will examine the possibility of uniaxial ordering to all orders in $\gamma$ and thus set $T=0$ in the ansatz Eq. (60). Note that in this case we must have $-1 / 2 \leq S \leq 1$. While within this ansatz positive $S$ corresponds to a normal nematic phase, the lower branch with negative $S$ implies excess material in the plane perpendicular to the nematic director and therefore represents a discotic phase.

We first define the rescaled free energy Eq. (55) as

$$
g(S)=\frac{12 \pi^{2} \beta \tilde{f}\left(\sigma_{U A}\right)}{\Lambda^{3}}
$$

Where $\sigma_{U A}$ is given by the Eq. (59) with $Q$ given by the uniaxial ansatz for Eq. (60) (thus with the term $T=0$ ); it can be evaluated explicitly and is given by

$$
\begin{aligned}
g(S)= & -2+2 \frac{\sqrt{3+\gamma(1-S)}}{\sqrt{3 \gamma S}} \cos ^{-1}\left(\frac{\sqrt{3+\gamma(1-S)}}{\sqrt{3+\gamma(1+2 S)}}\right) \\
& +\ln \left(\frac{1}{3}(3+\gamma(1+2 S))\right)+\frac{3 c}{1+2 S}+\frac{6 c}{1-S} .
\end{aligned}
$$

The first three terms represent the zero Matsubara frequency contribution to the electromagnetic field fluctuations. In the treatment of the full Lifshitz expression, for magnetically inactive media, these three terms would need to be summed over all the Matsubara frequencies as in Eq. (26).

We denote the first three terms in Eq. (62) as $g_{v d W}(S)$, i.e.

$$
g_{v d W}(S)=g(S, c=0) .
$$

As one of the main aims of this paper is to investigate the effect of the full n-body van der Waals interactions it is interesting to compare this term with $g_{v d W}^{(2)}(S)$, which corresponds to the pairwise approximation, and can be derived from Eq. (62) or the expression Eq. (36) truncated at the second order in $\gamma$, which gives

$$
g_{v d W}^{(2)}(S)=\frac{\gamma}{3}-\frac{\gamma^{2}}{90}\left(5+4 S^{2}\right)+\mathcal{O}\left(\gamma^{3}\right) .
$$

The negative coefficient of $S^{2}$ clearly demonstrates that the vdW interactions favor ordering at the pairwise level. However, one notes that at the pairwise level there is obviously no difference between the energy of nematic ordering (ordering along an axis) and discotic ordering (concentration in a plane) because the interaction is even in $S$. In the study of Edwards and Gupta ${ }^{1}$ it was found that within the ordered 
phase it is the nematic phase which has the lower free energy. The difference is due to the constraint part of $g(S)$, corresponding to the last two terms of Eq. (62), denoted by $g_{C O}(S)$, and given by

$$
g_{C O}(S)=g(S)-g_{v d W}(S)=\frac{9 c(1+S)}{(1+2 S)(1-S)} .
$$

This term favors nematic ordering over discotic ordering as can be seen by its Taylor expansion to fourth order in $S$

$$
g_{C O}(S)=9 c+18 c S^{2}-18 c S^{3}+54 c S^{4}+\mathcal{O}\left(S^{5}\right) .
$$

Here we can see how the symmetry of the vdW free energy between the nematic and discotic phases is broken by looking at the third order term in the interaction, corresponding to three body interactions. The total contribution to $g_{v d W}(S)$ to order $\gamma^{3}$ is then given by

$$
\begin{aligned}
g_{v d W}^{(3)}(S)= & \frac{\gamma}{3}-\frac{\gamma^{2}}{90}\left(5+4 S^{2}\right) \\
& +\frac{\gamma^{3}}{2835}\left(53+120 S^{2}+16 S^{3}\right)+\mathcal{O}\left(\gamma^{4}\right) .
\end{aligned}
$$

Therefore, for small $S$, where the term $S^{2}$ dominates, the three body interactions tend to increase the vdW interaction energy and thus they reduce the tendency toward ordering. Furthermore, we also see that the three body interaction favors discotic rather than nematic ordering due to the presence of a term proportional to $S^{3}$ with positive coefficient.

In Fig. 2 we show the comparison of $g_{v d W}(S)$ with its approximation at third order in $\gamma, g_{v d W}^{(3)}(S)$, for the value $\gamma=0.5$. We see that the approximation is very good at this value of $\gamma$, however the approximation tends to over estimate the zero Matsubara frequency vdW energy at all values of $S$.

As far as the phase diagram based on the assumption of nematic ordering is concerned, the rescaled free energy $g(S)$ for $\gamma=0.5$ is shown in Fig. 3. We see that when the value of $c$ is sufficiently lowered a first order nematic phase transition is induced. The transition occurs at $c^{*} \approx 0.000504$ and the order parameter $S$ jumps discontinuously from 0 to $S^{*} \approx 0.24$.

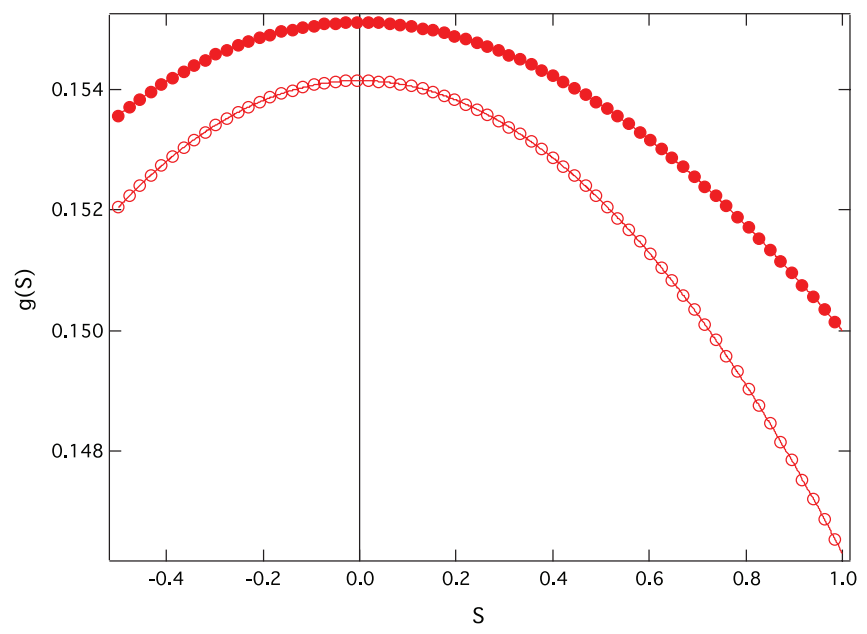

FIG. 2. The rescaled dependence of the zero frequency vdW energy on the order parameter, $g_{v d W}(S)=g(S, c=0)$ (Eq. (62)) for uniaxial ordering for the coupling parameter $\gamma=0.5$ compared with the expansions $g_{v d W}^{(3)}(S)$ of $g_{v d W}(S)$ to third order in $\gamma$ (Eq. (66)).

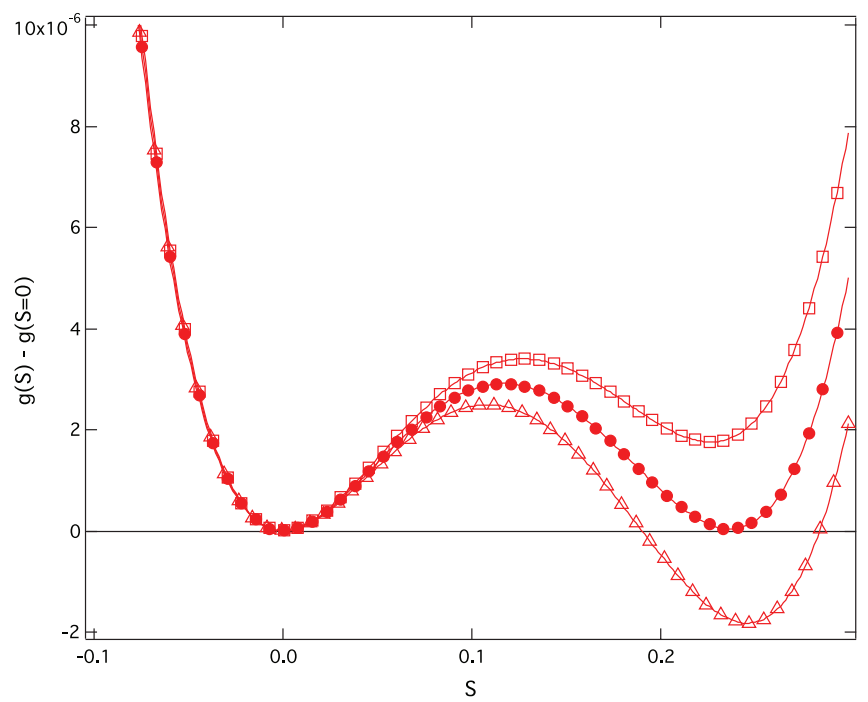

FIG. 3. The rescaled free energy difference (shifted for clarity by $g(S=0)$ ) $g(S)$ (Eq. (62)) for uniaxial ordering for the coupling parameter $\gamma=0.5$ for (from top to bottom) $c=0.000506$ (isotropic), $c=0.000504$ (coexistence between isotropic and nematic phases), $c=0.000502$ (nematic phase).

The complete dependence of the $c^{*}$ and $S^{*}$ on $\gamma$ is given on Fig. 4.

The way in which $c$ can be varied while keeping $\gamma$ constant is by changing the bare persistence length $l_{p}$ of the polymer. The isotropic nematic transition occurs at a critical value $l_{p}^{*}=3 v / 64 \pi^{2} \lambda^{3} c^{*}$. As $l_{p}^{*}$ is further increased, the order parameter $S$ increases and eventually tends to 1 .

In spite of the insights gained by this partitioning of the volume fraction dependence of $\gamma$ and $c$, it is necessary to investigate also the complete dependence of the free energy on the polymer density or equivalently its volume fraction. This dependence is hidden in $\gamma=\gamma(v)$, Eq. (35), and $c=c(v)$, Eq. (57).

Figure 5 shows the order parameter dependence of the total free energy Eq. (62) for three different values of the volume fraction $v$ contained in $\gamma=\gamma(v)$ and $c=c(v)$. The dielectric constants were taken as $\varepsilon_{\|}=4, \varepsilon_{\perp}=3$ and $\varepsilon_{w}=80$, which corresponds to a polymer with a hydrophobic core in an aqueous solution. The persistence length was taken as 2.5 in the units of the monomer size and the radius of the polymer was taken to be ten times the atomic size cutoff, $\lambda=10$.

For the chosen set of parameters the nematic transition occurs at $v=0.655$, where the minimum of the free energy is displaced from its disordered value $S=0$ to an ordered nematic state with $S \neq 0$. The critical concentration obviously depends on the exact values of the persistence length, the radius of the polymer and its dielectric anisotropy.

\section{DISCUSSION}

We have explored the consequences of orientationally dependent anisotropic vdW interactions on the ordering transition of semi-flexible polymers. A theory exact to all orders in the non-pairwise additive $\mathrm{vdW}$ interactions was derived and solved at the mean-field level. We have shown that there 


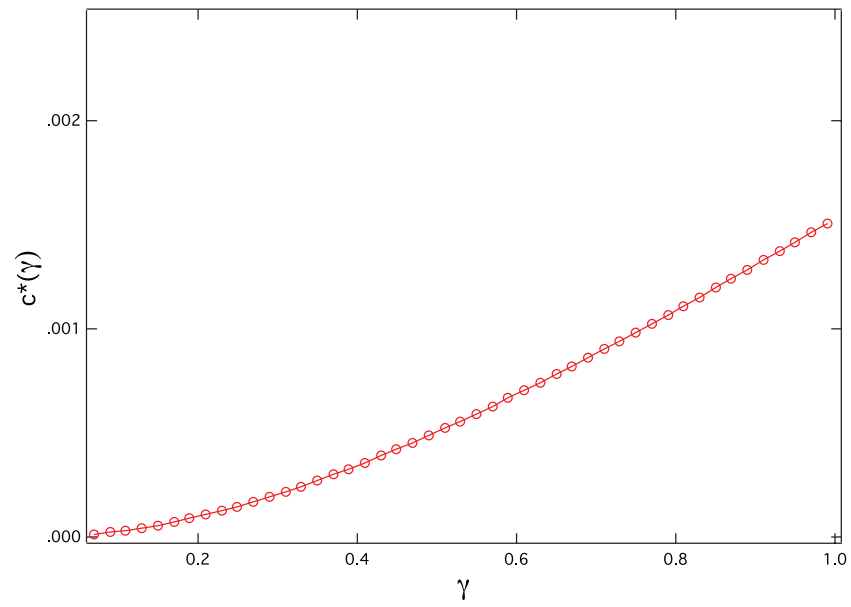

(a)

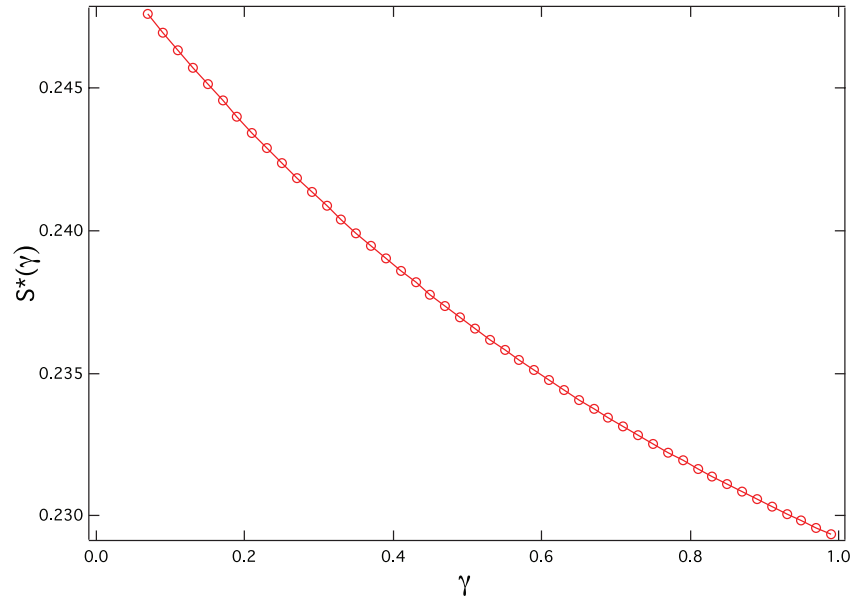

(b)

FIG. 4. The dependence of the $c^{*}$ and $S^{*}$ at the nematic transition on the interaction parameter $\gamma=\frac{\nu\left(\varepsilon_{\|}-\varepsilon_{\perp}\right)}{\varepsilon_{w}+\nu\left(\varepsilon_{\|}-\epsilon_{w}\right)}$.

exists an orientational ordering transition engendering a nematic polymer phase.

Though we limited ourselves to the zero Matsubara frequency term, the approach can be straightforwardly generalized to include the full anisotropic Lifshitz expression. ${ }^{16}$ In this case the free energy Eq. (62) needs to be rewritten as a full Matsubara frequency sum of the form

$$
\begin{aligned}
g(S, v)= & \sum_{n=0}^{\infty}\left(-2+2 \frac{\sqrt{3+\gamma_{n}(1-S)}}{\sqrt{3 \gamma_{n} S}} \cos ^{-1}\left(\frac{\sqrt{3+\gamma_{n}(1-S)}}{\sqrt{3+\gamma_{n}(1+2 S)}}\right)\right. \\
& \left.+\ln \left(\frac{1}{3}\left(3+\gamma_{n}(1+2 S)\right)\right)\right)+\frac{9 c(1+S)}{(1+2 S)(1-S)},
\end{aligned}
$$

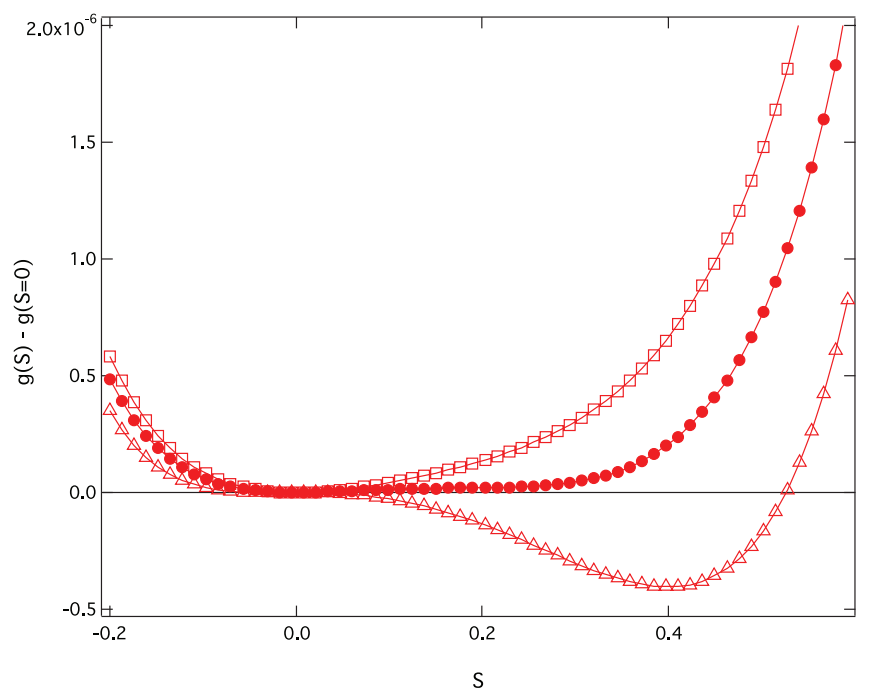

FIG. 5. The dependence of the free energy difference $g(S)-g(S=0)$ on the order parameter $S$ for various values of the volume fraction. Top to bottom $v=0.63,0.655,0.68$. We have taken $\varepsilon_{\|}=4, \varepsilon_{\perp}=3$, and $\varepsilon_{w}=80$ and $\mathcal{C}=2 \times 10^{-6}$. The value for $\mathcal{C}$ corresponds to $l_{b}=2.5$ and $\lambda=10$. where now

$$
\begin{aligned}
\gamma_{n}=\gamma\left(\nu, l \xi_{n}\right) & =\frac{v\left(\varepsilon_{\|}\left(l \xi_{n}\right)-\varepsilon_{\perp}\left(l \xi_{n}\right)\right)}{\varepsilon_{w}\left(l \xi_{n}\right)+v\left(\varepsilon_{\|}\left(l \xi_{n}\right)-\varepsilon_{w}\left(l \xi_{n}\right)\right)} \quad \text { and } \\
c & =\frac{3 \pi v}{8 \Lambda^{3} a^{2} b l_{p}}=\frac{3}{64 \pi^{2} \lambda^{3}} \frac{v}{l_{p}}
\end{aligned}
$$

Depending on the dielectric anisotropy $\varepsilon_{\|}\left(1 \xi_{n}\right)-\varepsilon_{\perp}\left(1 \xi_{n}\right)$ at every Matsubara frequency, each term in the sum makes a contribution to the total ordering free energy. This means that the vdW part of the free energy in general increases and therefore displaces the ordering transition towards smaller densities. One should note that the overall density dependence of Eq. (67) becomes quite complicated in this case as it has a component (the first three terms) which depends on the frequency dependent dielectric response, and a component that does not (the last term coming from the constrained KP polymer free energy). We also note that the temperature dependence of the free energy, upon the inclusion of the non-zero Matsubara frequencies, becomes much more complex. However, detailed computations of the van der Waals interaction in aqueous solutions show that the zero frequency Matsubara term gives more then $50 \%$ of the contribution to the total vdW interaction. ${ }^{22}$ This is a particularity of water dielectric dispersion, stemming from its very large dipole moment and high static dielectric response. The order of magnitudes predicted in this paper based on solely the zero-frequency Matsubara frequency should give a good estimate of the effect of $\mathrm{vdW}$ interactions on ordering transitions. However, the frequency dependent components will play a significant role, and possibly recent experimental developments will allow a determination of their behavior. ${ }^{15}$

The total free energy thus contains two separate contributions: the Matsubara sum corresponding to the anisotropic $\mathrm{vdW}$ interactions and the last term stemming from the "constraint" free energy, that connects the dielectric anisotropy of the polymer chain with its configuration in space. It is the last term that cannot be evaluated precisely because it 
contains a spatial cutoff $\Lambda$ due to the continuum nature of the electrostatic field description. This is not a major impediment regarding the scaling properties and the existence of the transition, however, it introduces questions regarding the exact numerical values for the transition density. Based on the estimates made here, we conclude that the polymer does not have to be very stiff in order to show the vdW driven orientational ordering transition.

The mean-field approach outlined here for the case of orientationally dependent vdW interactions can of course be easily extended and applied to other cases. The coupling of the zero frequency vdW interaction to an electrolyte in the solvent would be of particular interest to analyze.

\section{ACKNOWLEDGMENTS}

D.S.D. acknowledges support from the Institut Universitaire de France. R.P. acknowledges support from ARRS through the program P1-0055 and the research project J1-4297 as well as the University of Toulouse for a one month position of Professeur Invité.

\footnotetext{
${ }^{1}$ A. M. Gupta and S. F. Edwards, J. Chem. Phys. 98, 1588 (1993).

${ }^{2}$ M. W. Davidson, T. E. Strzelecka, and R. L. Rill, Nature (London), 331, 457 (1988); F. Livolant, A. M. Levelut, J. Doucet, and J. P. Benoit, ibid. 339, 724 (1989).
}

${ }^{3}$ R. Podgornik, H. H. Strey, K. Gawrisch, D. C. Rau, A. Rupprecht, and V. A. Parsegian, Proc. Natl. Acad. Sci. U.S.A. 93, 4261 (1996).

${ }^{4}$ F. Livolant, Phys. A 176, 117 (1991).

${ }^{5}$ R. Podgornik, H. H. Strey and V. A. Parsegian, "Colloidal DNA," Curr. Opin. Colloid Interface Sci. 3, 534 (1998).

${ }^{6}$ Y. M. Evdokimov, Liquid Crystalline Dispersion and DNA Nanoconstructions/Zhidkokristallicheskie Dispersii $i$ Nanokonstruktsii DNK, (Radiotekhnika, Moscow, 2008).

${ }^{7}$ M. Manchester and N. F. Steinmetz, Viruses and Nanotechnology, Current Topics in Microbiology and Immunology, 1st ed. (Springer, 2008).

${ }^{8}$ L. Onsager, Ann. N.Y. Acad. Sci. 51, 627 (1949).

${ }^{9}$ A. N. Semenov, A. R. Khokhlov, Sov. Phys. Usp. 31, 988 (1988).

${ }^{10}$ G. Ronca and D. Y. Yoon, J. Chem. Phys. 76, 3295 (1982).

${ }^{11}$ A. Tkachenko and Y. Rabin, Macromolecules 28, 8646 (1996).

${ }^{12}$ R. Podgornik, Phys. Rev. E 52, 5170 (1996).

${ }^{13}$ R. H. French, V. A. Parsegian, R. Podgornik et al., Rev. Mod. Phys. 82, 1887 (2010).

${ }^{14}$ J. B. MacNaughton et al., J. Phys. Chem. B 110, 15742 (2006).

${ }^{15}$ W. Y. Ching (private communication).

${ }^{16}$ V. A. Parsegian, $v d W$ Forces (Cambridge University Press, Cambridge, 2005); M. Bordag, G. L. Klimchitskaya, U. Mohideen, and V. M. Mostepanenko, Advances in the Casimir Effect (Oxford University Press, New York, 2009).

${ }^{17}$ V. A. Parsegian and G. H. Weiss, J. Adhes. 3, 259 (1972).

${ }^{18}$ J. N. Munday, D. Iannuzzi, Y. Barash, and F. Capasso, Phys. Rev. A 71, 042102 (2005).

${ }^{19}$ Yu. S. Barash, Izv. Vyssh. Uchebn. Zaved. Radiofiz. 21, 163 (1978).

${ }^{20}$ A. Siber, R. F. Rajter, R. H. French, W. Y. Ching, V. A. Parsegian, and R. Podgornik, Phys. Rev. B 80, 165414 (2009).

${ }^{21}$ A. Siber, R. F. Rajter, R. H. French, W. Y. Ching, V. A. Parsegian, and R. Podgornik, J. Vac. Sci. Technol. B 28, C4A17 (2010).

${ }^{22}$ J. Mahanty and B. W. Ninham, Dispersion Forces (Academic, London, 1976). 